\title{
Practical Applications of Synthetic Aperture Imaging
}

Nikolov, Svetoslav Ivanov; Kortbek, Jacob; Jensen, Jørgen Arendt

\section{Published in:}

Proceedings of IEEE International Ultrasonics Symposium

Link to article, DOI:

10.1109/ULTSYM.2010.0086

Publication date:

2010

Document Version

Early version, also known as pre-print

Link back to DTU Orbit

Citation (APA):

Nikolov, S. I., Kortbek, J., \& Jensen, J. A. (2010). Practical Applications of Synthetic Aperture Imaging. In Proceedings of IEEE International Ultrasonics Symposium (pp. 350-358). IEEE.

https://doi.org/10.1109/ULTSYM.2010.0086

\section{General rights}

Copyright and moral rights for the publications made accessible in the public portal are retained by the authors and/or other copyright owners and it is a condition of accessing publications that users recognise and abide by the legal requirements associated with these rights.

- Users may download and print one copy of any publication from the public portal for the purpose of private study or research.

- You may not further distribute the material or use it for any profit-making activity or commercial gain

- You may freely distribute the URL identifying the publication in the public portal

If you believe that this document breaches copyright please contact us providing details, and we will remove access to the work immediately and investigate your claim. 


\title{
Practical Applications of Synthetic Aperture Imaging
}

\author{
Svetoslav Ivanov Nikolov*, Jacob Kortbek* and Jørgen Arendt Jensen ${ }^{\dagger}$ \\ *BK Medical Aps, Mileparken 34, 2730 Herlev, Denmark, Email:sin@bkmed.dk \\ ${ }^{\dagger}$ Technical University of Denmark, Center for Fast Ultrasound Imaging, 2800 Kgs. Lyngby, Denmark
}

\begin{abstract}
Synthetic aperture imaging has been a focus of research for almost 3 decades. The research carried out at the Center for Fast Ultrasound Imaging has demonstrated that synthetic aperture focusing not only can be used in-vivo, but that it also yields superior B-mode and blood flow images.

In the last years synthetic aperture focusing has moved from the lab to commercial products. The implementations vary in their scope and purpose. Some scanners use synthetic aperture imaging to improve the detail and contrast resolution of the system. Others to increase the image uniformity. Yet others use synthetic aperture acquisition to achieve high frame rates and superior flow estimations. On the other end of the scale are the systems that utilize synthetic aperture techniques to reduce the data rate and take advantage of modern computer hardware.

Retrospecitve transmit beamformation, zone sonography, and multiple angle flash imaging are just a few of the names used to describe the commercial implementations of synthetic aperture focusing. Although they sound like different algorithms, they are the same in their core, as revealed in this paper.
\end{abstract}

\section{INTRODUCTION}

We start our journey in with a short history of beamforming in which we explore the different challenges that designers face. We look at basic system parameters like resolution, frame rate, and image uniformity in the next section. The following sections III, IV, and V address each of the challenges and show how resolution can be improved by synthetic receive aperture, image uniformity by synthetic transmit aperture with virtual sources, and the frame rate by sparse synthetic transmit aperture. Finally we look at the exciting opportunities for precise flow estimation in section VII.

\section{SHORT HISTORY OF BEAMFORMING}

Ultrasound imaging is a pulse-echo modality, in which an acoustic pulse is transmitted in the tissue. As the pulse propagates some of the energy is reflected and scattered back to the transducer.An image is formed by focusing a beam in the imaged direction.

The transmission has traditionally been focused and delayand-sum beamforming has been applied on the signals received by a transducer array as shown in Fig. 1. A line in the image $s(t)$ is formed by applying delays $\tau_{j}$ on the received signals $g_{j}(t)$. The echoes are apodized by applying weight coefficients $a_{j}$. Both, the apodization coefficients, and the delays are a function of depth (time) leading to a dynamic receive beamforming. The process is expressed mathematically

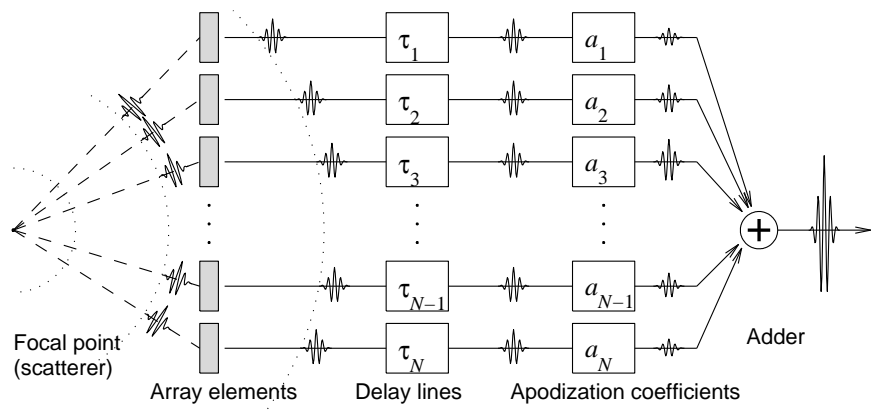

Fig. 1. Delay-and-sum beam forming (from [1]).

as

$$
s(t)=\sum_{j=1}^{N_{r}} a_{j}(t) g_{j}\left(t-\tau_{j}\right),
$$

where $N_{r}$ is the number of active receive elements. The area spanned by the transmit and receive elements represents the transmit and receive apertures, respectively. For many applications this processing is sufficient, but there are a number of areas, where it is desirable to overcome some inherent limitations due the delay-and-sum beamformer, combined with focused transmits:

\section{A. Performance/cost tradeoff}

The lateral resolution of the system, measured as the full width of the beam at half maximum $\left(b_{F W H M}\right)$ can be approximated roughly as:

$$
b_{F W H M} \sim \lambda f_{\#}=\lambda \frac{F}{D}
$$

where $\lambda$ is the wavelength, $F$ is the focus depth, and $D$ is the size of the aperture. The value $f_{\#}$ is known as the "f-number" and is defined as the ratio between the focal distance and the aperture size.

The performance of a system is directly proportional to the size of the active aperture, which translates into a large number of (primarily receive) channels. The cost of the system is also directly proportional to the number of channels. Ideally we would like to create a large imaging aperture without an increase in the number of processing channels. 

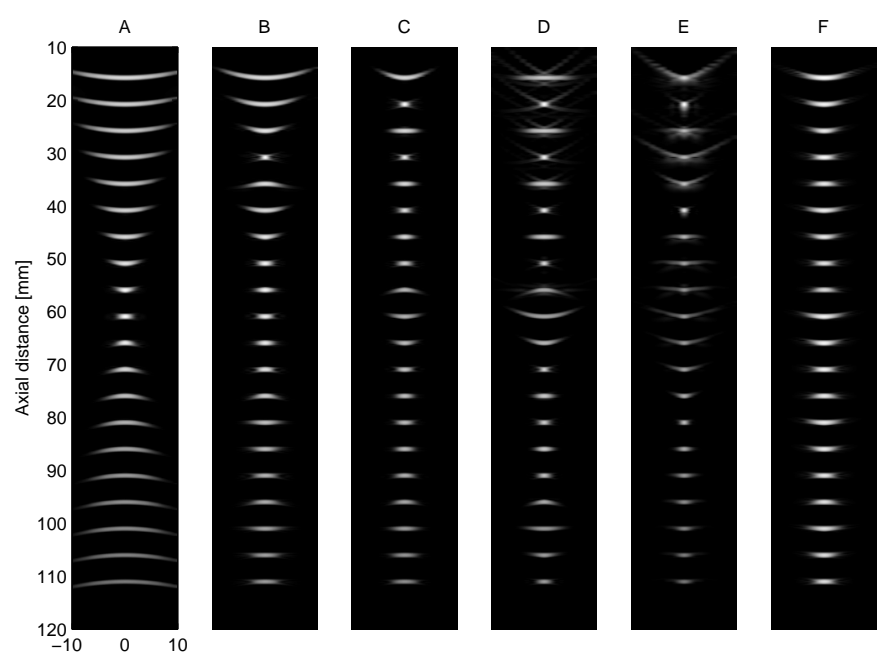

Lateral distance $[\mathrm{mm}]$

Fig. 2. Example point spread functions. A - one focal zone, B - 6 receive focal zones, C - 6 zones receive xmt and rcv, D - 128 elem, 4 zones xmt, F - 128 elem, xmt $f_{\#}=4, \operatorname{rcv} f_{\#}=2$.

\section{B. Frame rate}

Sound waves propagate with a finite speed in tissue, which on average is assumed to be

$$
c=1540 \mathrm{~m} / \mathrm{s} \text {. }
$$

The number of lines in the image must meet the sampling criteria and is often rather large - up to 200 - 400 lines. The time for acquiring a single line is then:

$$
T_{p r f}=\frac{2 z_{\max }}{c},
$$

where $z_{\max }$ is the maximum scan depth. Let the number of lines in the image be $N_{l}$. The time for acquisition of a single frame is $T_{p r f} \times N_{l}$. The frame rate is then

$$
f_{f r}=\frac{c}{2 N_{l} z_{\max }} .
$$

Assuming a typical abdominal scan with a curved linear array with 192 elements, and a line density of 1.5 lines per element we have $N_{l}=288$. The scan depth is typically up to $15 \mathrm{~cm}$, and in larger patients $20-25 \mathrm{~cm}$, which gives frame rates in the range of

$$
f_{f r} \sim 12-17 \quad \frac{\text { frames }}{\mathrm{sec}} .
$$

Color flow mapping requires a number of shots - typically 4 to 16 - for every beam in which the flow is estimated, which further reduces the frame rate.

To overcome this limitation, parallel beamforming is used, which introduces a problem with image uniformity.

\section{Image uniformity}

Fig. 2 shows the point spread function of a ultrasound system as a function of depth. It can be seen that it is spatially variant. The focusing capabilities of the system deteriorate

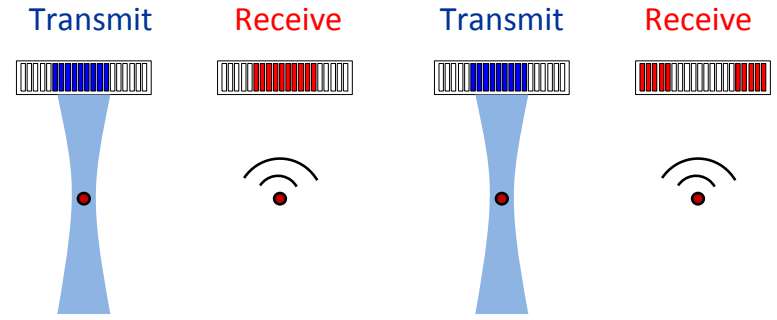

Fig. 3. Synthetic receive aperture imaging.

away from the transmit focus. Many systems overcome this problem by using $N_{f}$ transmit foci, which reduces the frame rate by a factor $N_{f}$. To decrease the overall acquisition time, systems may utilize a varying acquisition duration (shortest acquisition time for the closest focal zone, longer for the next, etc.)

Parallel beamforming can be used to increase the frame rate. When more than 2 beams are formed in parallel, artifacts in the image appear. The main reason is that beams lying close to the center of the transmitted beam have higher levels than beams at the edges. Simple gain does not suffice, since there is a variation in signal-to-noise ratio as well as in propagation time (phase).

\section{ENLARGING THE RECEIVE APERTURE}

A common technique used today is the use of synthetic receive aperture [2], [3]. Fig. 3 illustrates the process of synthetic receive aperture focusing. In the example, the same transmit aperture is used twice to scan a line in the image. Two different apertures are used in receive. Which receive elements that are active during the first acquisition, and which during the second, is in principle irrelevant. Since data are acquired over a number of emissions, motion artifacts are present. Motion compensation is desirable in the case of fast motion, and has been investigated by Nock and Trahey [2], [3]. Two cases have been considered grouping the elements in right/left subapertures and grouping them in odd/even (interleaved) subapertures. The correlation between the beamformed lines from the two acquisitions is higher when the elements of the subapertures are interleaved. This allows for motion estimation between the two firings and subsequent motion compensation.

In practice, however, the choice of subapertures is dictated by how multiplexors are connected to the transducer elements, and their switching speed. A more common method is to divide the elements in inner and outer sub-apertures, and is shown in Fig. 3. Usually the apodization function is dynamic, and attempts to maintain a constant $f_{\#}$. In the start only the elements closest to the center of the transmit beam are used in receive beam sum. Inner elements are needed immediately after the emission. As the aperture grows more the elements from the outer subaperture get activated. This relaxes the requirements for switch times of the multiplexors compared to the odd/even or the interleaved cases.

Fig. 4 shows the maximum number of receive elements for a curved abdominal probe. The upper subplot shows the 


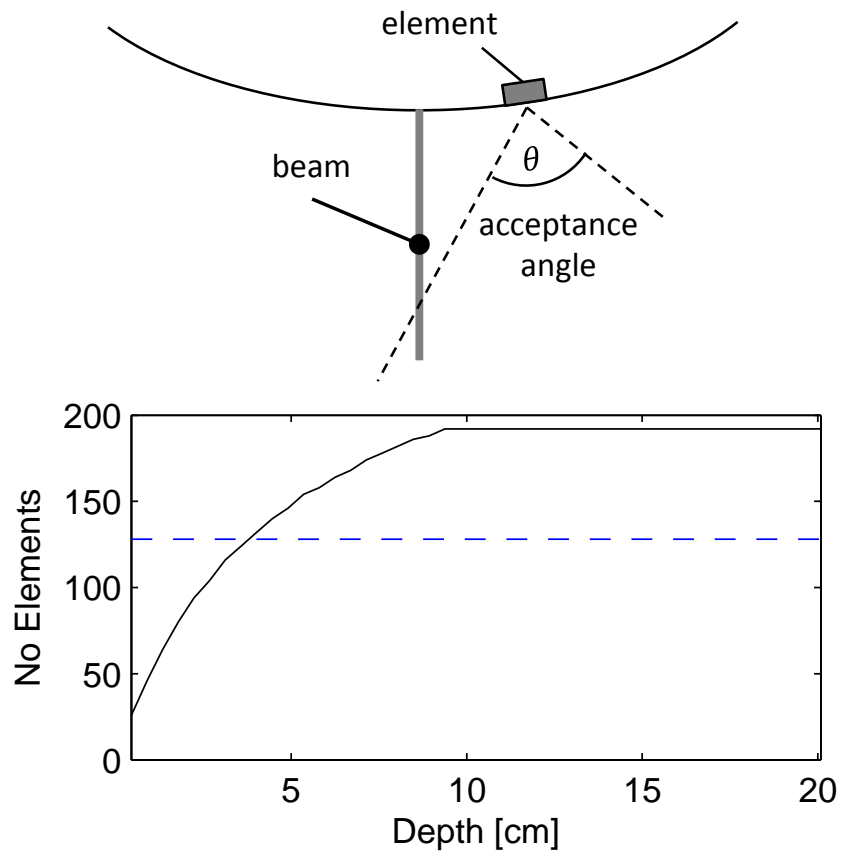

Fig. 4. Maximum number of used elements as a function of depth. Geometry to determine the number of elements is shown in the upper subplot, and the maximum number of used elements in the bottom plot. The dashed line shows the limit of 128 elements. The ultrasound probe is a curved abdominal array with 192 elements, center frequency of $4 \mathrm{MHz}$, and a radius of curvature of $61.5 \mathrm{~mm}$, and a pitch of $330 \mu \mathrm{m}$.

geometry used to determine the number of elements that can be used in receive. An element can be used when the samples in the beam are inside the acceptance angle of the respective element.

The acceptance angle $\theta$ is found from the radiation pattern of the element, also known as element factor. $\theta$ is the angle at which the radiation pattern $A(\theta)$ drops below a desired level. A good value lies between $30 \%$ and $50 \%$ of the maximum element sensitivity. A useful approximation for the normalized radiation pattern is given by [4]

$$
A(\theta)=\frac{\sin (\pi \omega / \lambda \sin \theta)}{\pi \omega / \lambda \sin \theta} \cos \theta
$$

where $\lambda=c / f_{0}$ is the wavelength, $\omega=2 \pi f_{0}$, and $f_{0}$ is the center frequency.

As it can be seen, even for a curved array it is possible to use all elements in the probe. Many scanners have less than 128 simultaneously active channels (often only 64) and a receive aperture is synthesized to improve image resolution, contrast, and penetration depth.

\section{CREATING AN UNIFORM IMAGE}

Most phased array systems use a fixed transmit focus and dynamic receive focusing. As shown in Fig. 2, the fixed transmit focus imposes limitations on the quality of the image away from the transmit focus. A practical approach was suggested in 1995 by Freeman, Li and O'Donnel [5] and named "retrospective dynamic transmit focusing". The approach uses

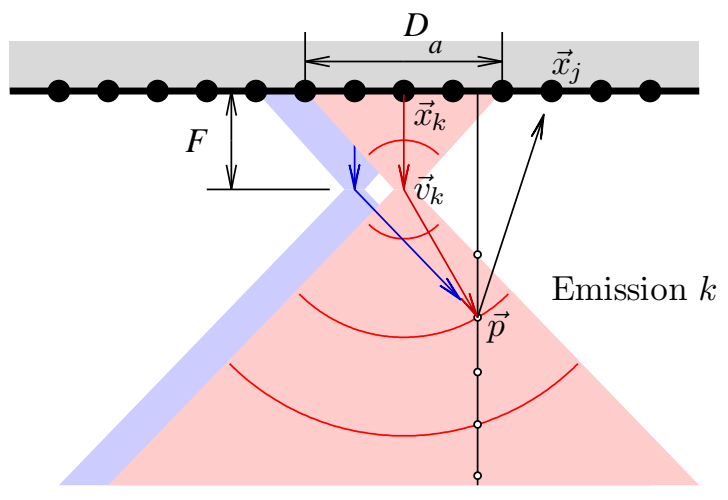

Fig. 5. RF channel data from several emissions can be used to beamform the same image line.

the fact that a beam spans several image lines. In the original work, the authors suggest to use a depth-dependent filter bank of inverse filters which are applied on the scan lines in a lateral direction.

This approach has been further refined by a number of researchers as synthetic transmit aperture with focused virtual sources [6]-[9]. A similar approach is used in commercial scanners [10].

The geometry is illustrated in Fig. 5. It uses a simple geometrical approximation of the wave propagation. The transmitted beam is assumed to converge at the transmit focus. For emission $k$, the transmit focus is located at $\vec{v}_{k}$. The focal point is considered as a virtual source, which transmits spherical waves. To beamform a point $\vec{p}$, we need to coherently sum the echoes coming to the transducer surface. The total propagation time from the origin of emission $k$ to the beamformed point $\vec{p}$ and back to a transducer element with coordinates $\vec{x}_{j}$ is:

$$
t=\frac{1}{c}\left(\left|\vec{v}_{k}-\vec{x}_{k}\right|+\left|\vec{p}-\vec{v}_{k}\right|+\left|\vec{x}_{j}-\vec{p}\right|\right)
$$

Beamformers operate either with relative delays (one channel relative to another) or with absolute propagation times. In the case of synthetic transmit aperture the propagation time can be divided in transmit and receive propagation time [9]:

$$
t=t_{x}+t_{r}
$$

The receive propagation time is

$$
t_{r}=\frac{\left|\vec{x}_{j}-\vec{p}\right|}{c}
$$

It is independent of the transmission and do not need to be changed from acquisition to acquisition [11]. The transmit propagation time is

$$
t_{x}=\frac{1}{c}\left(\left|\vec{v}_{k}-\vec{x}_{k}\right|+\left|\vec{p}-\vec{v}_{k}\right|\right)
$$

It contains 2 components: the first from the transducer surface to the focal point $\vec{v}_{k}$, and the second from the focal point to the imaged point $\vec{p}$. Most systems will have identical transmissions, and the first component will be constant $t_{v}$ :

$$
t_{v}=\frac{\left|\vec{v}_{k}-\vec{x}_{k}\right|}{c} .
$$




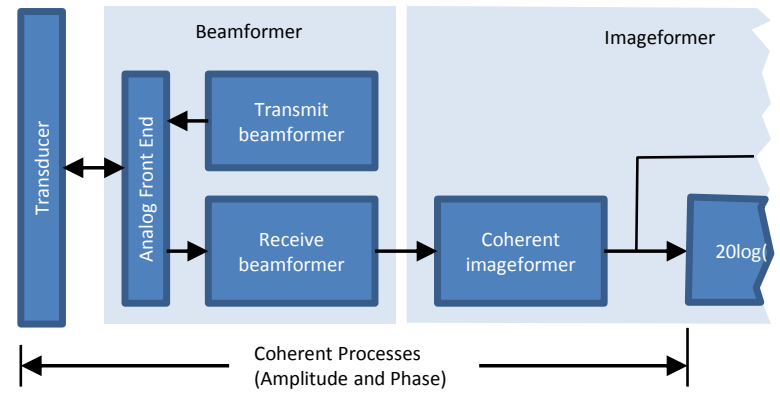

Fig. 6. Conceptual block diagram of imaging engine capable of synthetic transmit (and/or receive) aperture focusing (from [12]).

The beamforming of a line $s(t)$ becomes

$$
s(t)=\sum_{k=1}^{N_{x}} \sum_{j=1}^{N_{r}} a_{k}(t) a_{j}(t) g_{k j}\left(t-\tau_{x}(k, t)-\tau_{r}(j, t)\right)
$$

where $\tau_{x}$ is the relative transmit delay which varies with the transmission $k$ and $\tau_{r}(j)$ is the relative receive delay.

There are different architectural possibilities to implement this type of synthetic transmit aperture beamforming and Fig. 6 shows one of them. The first stage is a beamformer, which receives channel data and performs a delay and sum beamforming. Ideally, this is the only stage doing coherent processing, but often practical limitations necessitate the distribution of processing over several units.

The second processing unit is called "coherent image former", and this is the place where accumulation of partial beamsums from different emissions occurs. The processing from (11) can be divided between the two units as:

$$
\begin{aligned}
s_{k}(t) & =\sum_{j=1}^{N_{r}} a_{j}(t) g_{k j}\left(t-\tau_{x}(k, t)-\tau_{r}(j, t)\right) \\
s(t) & =\sum_{k=1}^{N_{x}} a_{k}(t) s_{k}(t)
\end{aligned}
$$

or as

$$
\begin{aligned}
s_{k}(t) & =\sum_{j=1}^{N_{r}} a_{j}(t) g_{k j}\left(t-\tau_{r}(j, t)\right) \\
s(t) & =\sum_{k=1}^{N_{x}} a_{k}(t) s_{k}\left(t-\tau_{x}(k, t)\right)
\end{aligned}
$$

Notice that the coherent image former can be used to perform both synthetic transmit, and synthetic receive focusing. The image former needs a buffer large enough to store a portion of the image corresponding to the maximum extent of the transmit beam. According to reference [12], modern commercial scanners can beamform up to 64 beams in parallel.

The use of synthetic transmit aperture beamforming has a large impact on the image quality as shown in Fig. 7. The scan has been performed using a 128-element linear array with a center frequency $f_{0}$ of $7.5 \mathrm{MHz}$, pitch of $208 \mu \mathrm{m}$. The transmit focus is placed at $40 \mathrm{~mm}$. The number of active
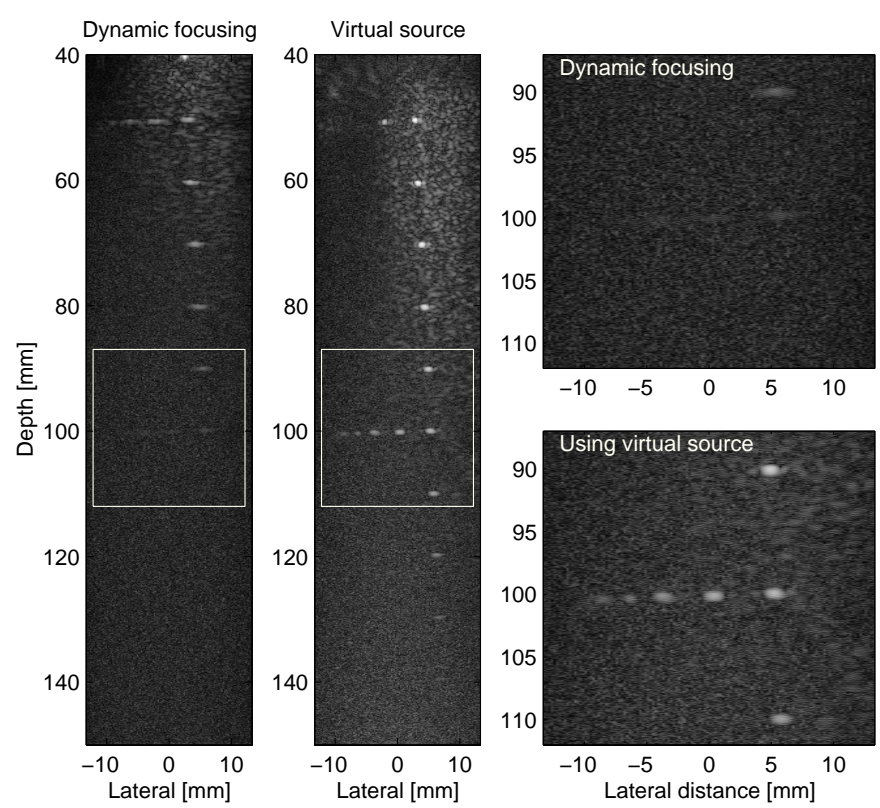

Fig. 7. B-mode images of a tissue-mimicking phantom (from [9]). The two images are created from the same receive data, but in the right one synthetic transmit focusing has been applied.

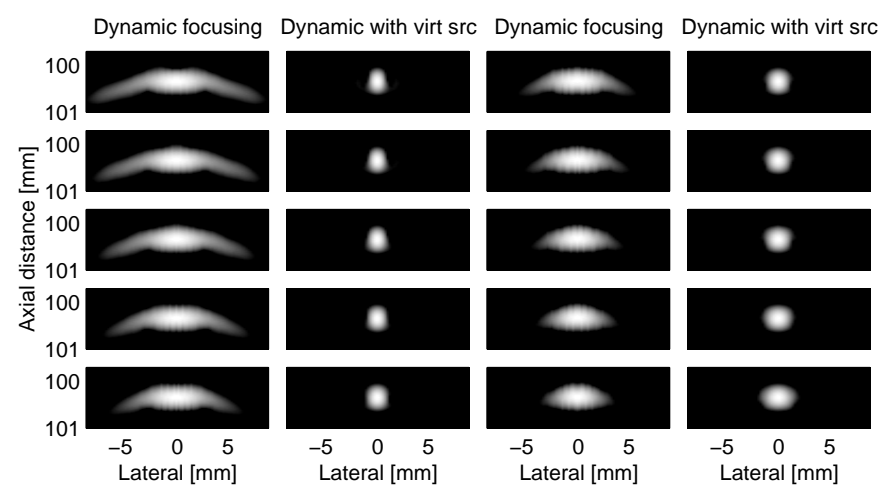

Fig. 8. Simulated B-mode images of a scatterer placed $100 \mathrm{~mm}$ below the transducer. The transmit focus is varied between $30 \mathrm{~mm}$ and $75 \mathrm{~mm}$ at steps of $5 \mathrm{~mm}$. In columns 1 and 3 the focusing in receive is dynamic. In columns 2 and 4 the dynamic receive focusing is combined with synthetic transmit aperture focusing based on a virtual source element.

receive elements is 64 . The same data set has been used to create images with and without synthetic transmit focusing. The result of applying synthetic transmit aperture focusing is an increased signal-to-noise ratio, higher resolution and penetration depth.

Fig. 8 shows the achievable resolution for a scatterer placed $100 \mathrm{~mm}$ from the transducer surface as a function of the placement of transmit focus. As expected, the closer the transmit focus is to the point scatterer, the better the resolution using only dynamic receive focusing. Combining the receive focusing with a synthetic transmit aperture, however exhibits the opposite trend - the further the focus from the transducer surface, the worse the resolution. The reason for this is that the size of the transmit aperture $D_{a}$ is fixed, and increasing 


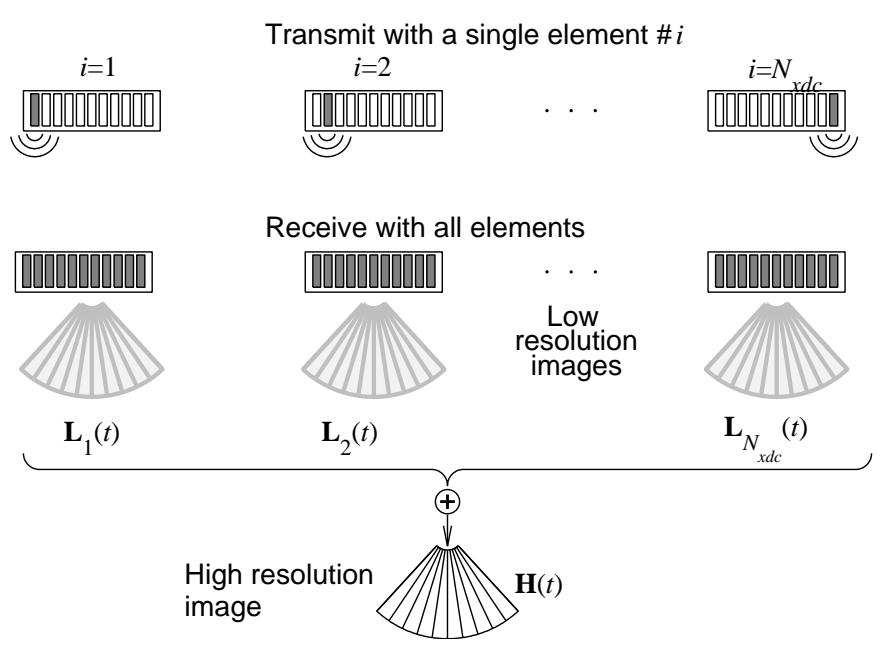

Fig. 9. Synthetic transmit aperture imaging (from [15]).

the focal distance $F$ results in smaller acceptance angle for the transmit aperture, hence a smaller transmit aperture can be synthesized (see Fig. 5). A useful rule of the thumb can be that the achievable resolution is equal to the resolution at the transmit focus. A more extensive study of how much the geometric approximation of virtual sources matches the real beam profile, and the influence of the opening angle can be found in [1], [9], [13], [14]

\section{HIGH FRAME RATE}

Sparse synthetic transmit aperture imaging has the potential for high frame rate imaging by decoupling the transmissions from the image size [16]-[21].

Fig. 9 illustrates synthetic transmit aperture focusing. The principle of synthetic transmit aperture imaging is to acquire backscattered data with different phase and amplitude information which will allow to reconstruct the image using, for example, delay-and-sum beamforming. Fig. 9 shows the use of spherical wave transmission to scan the region. First a spherical wave is created such as its origin is at the one end of the transducer. The wave propagates in the whole region under investigation and is back scattered to the transducer. Then the acquisition is repeated, but this time the origin of the spherical wave is positioned at another spatial location. The process is repeated until the whole transmit aperture is spanned by the centers of origin of the spherical waves. At every emission a full image is created by delay and sum beamforming. These images have a low resolution, since beamforming is performed only in receive. Combining a number of low resolution images creates a high-resolution image.

A certain insight can be gained by inspecting the process of creating a high-resolution image. Consider Fig. 10. It shows how an image of a single point scatterer is created using 5 emissions (only 4 actually plotted). First the left-most element sends a wave. Upon reception a low-resolution image $L^{(1)}$ is formed. The high-resolution image $H^{(n)}$ at step $n$ is formed
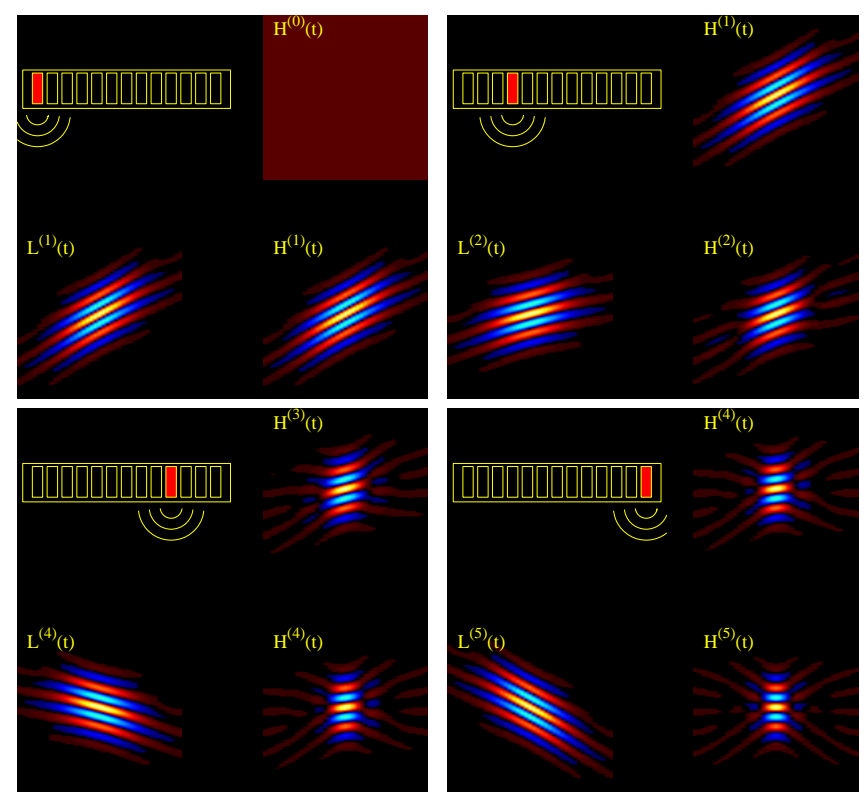

Fig. 10. Illustration of the beamforming process.

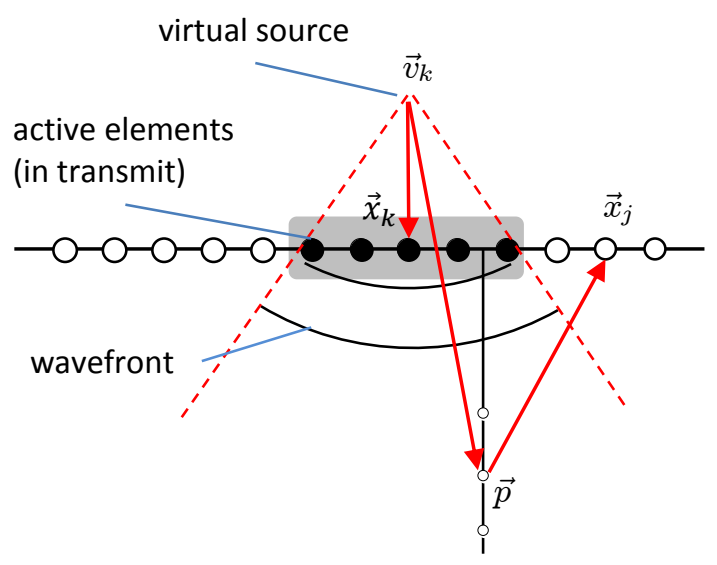

Fig. 11. Propagation path to be used in delay calculations using a spherical wave transmission.

by accumulating the low-resolution images

$$
H^{(n)}=H^{(n-1)}+L^{(n)}
$$

So after the first emission, $H^{(1)}=L^{(1)}$. The image is tilted towards the origin of the emission. After the second emission a second low resolution $L^{(2)}$ image is created. The point spread function at the point scatterer is now rotated at a different angle and is facing the direction of the second emission. The center of rotation is the beamformed point. Adding $L^{(1)}$ to $H^{(1)}$ means that the echoes at the center of the point are summed in phase and hence amplified. The echoes at the edges of the point spread function are added out of phase and hence attenuated. Repeating the acquisition, so that the whole aperture is covered, results in an image of the point scatterer that has much higher lateral resolution than the individual lowresolution images. This process is explained more rigorously in [1]. 


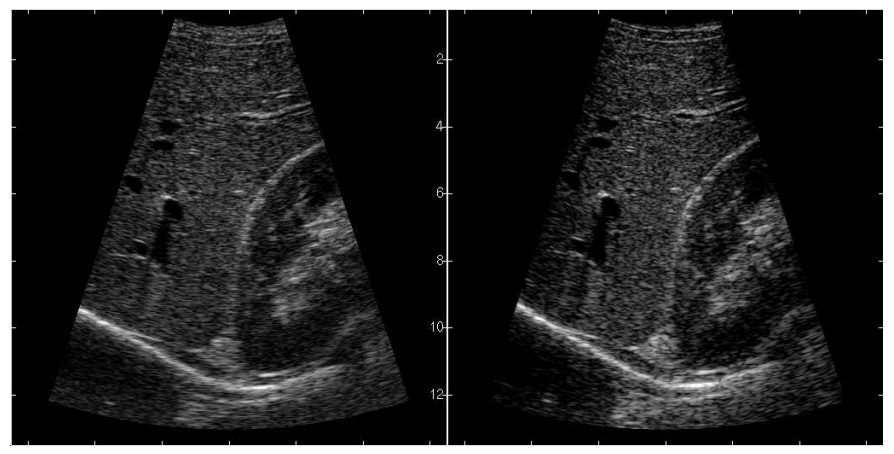

Fig. 12. Side-by-side images from a pre-clinical study performed by Pedersen, Gammelmark and Jensen [22], [23]

In practice multiple elements are used to create a spherical wave. Instead of using the center of the active aperture as the origin of the transmission, it is better to use the propagation path from a virtual source placed "behind" the transducer surface to the imaged point and back to a receive element as shown in Fig. 11. The propagation path consists of the same components as in (6), but the time from the aperture to the virtual source must be subtracted:

$$
t=\frac{1}{c}\left(\left|\vec{p}-\vec{v}_{k}\right|+\left|\vec{x}_{j}-\vec{p}\right|-\left|\vec{v}_{k}-\vec{x}_{k}\right|\right)
$$

The image quality of synthetic transmit aperture has been evaluated in a pre-clinical study by Pedersen, Gammelmark, and Jensen [22], [23]. The double-blinded study revealed that the synthetic aperture images have better image quality than conventional dynamically focused images.

Resolution is dependent on the size of the transmit aperture and a sparse transmit aperture can significantly increase the frame rate.

Presently, only research systems have been built that implementing synthetic transmit aperture focusing [11], [11], [17], [24]-[28]. The system designed by Jensen et al. [26] partitions the beamformation differently compared to the system depicted in Fig. 6. The beamformer is split in a number of physical units, each processing data from up to 16 channels. Each unit creates "partial" high resolution images, which are accumulated over all emissions that go into the high resolution image, but only for the receive channels that are physically connected to the beamforming unit. After all emissions have been performed, then the partial high resolution images are summed in a second stage. The beamforming units are implemented in FPGAs. The internal RAM is used as a cache. The total image is stored in external DRAM. A portion of the image is read into the FPGA's buffers, the channel data are added to the buffer according to a propagation delay calculated either by (6) or by (17). Then the cache is stored back to the external RAM and another portion of the image is processed.

A similar approach has been used in a commercial system developed by Zonare (420 N. Bernardo Ave, Mountain View, CA 94043-5209), in which the processing is handled by digital signal processors [29], [30]. Few transmissions per zone relieve the requirements for processing power, while maintaining

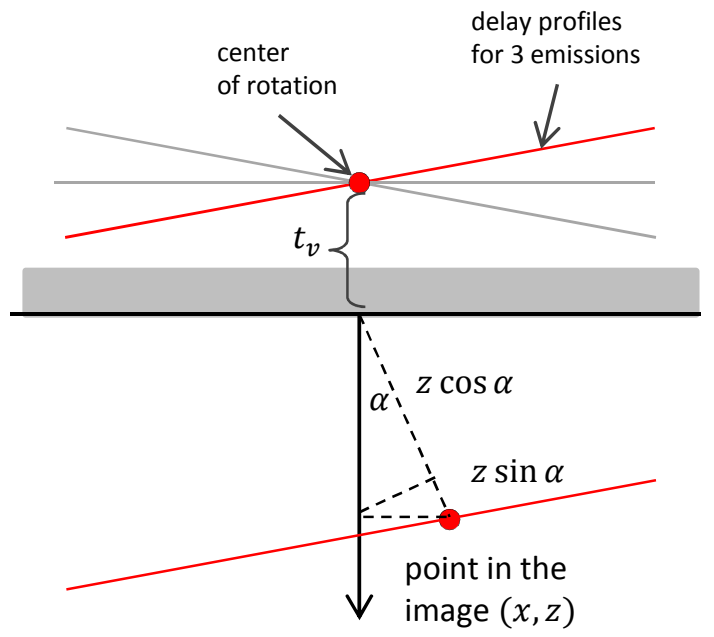

Fig. 13. Using plane waves to create an image.

image quality and frame rate comparable to conventional systems.

Looking at Fig. 10 one can see that the essence of creating a high-resolution image is to illuminate a point scatterer from different directions and add the return echoes coherently. What is important is to be able to model the wavefront of the impinging wave. Virtual sources provide a simple geometric model for focused or unfocused waves. One type of unfocused waves are the plane waves. Plane waves have been used as a method alternative to sparse synthetic transmit aperture to achieve high frame rates [31]-[33].

Normal delay and sum beamforming can be used also with plane wave imaging. The receive delays are calculated as in focused transmit imaging. The transmit delays must take into consideration the propagation of the plane wave as shown in Fig. 13. A convenient method is to add a constant time offset $t_{v}$ to the delays used to generate the plane wave. This means that the plane wavefronts are rotated about a point placed "behind" the transducer. The propagation time from the center of the transducer array to a point in the image placed at coordinates $(x, z)$ (see Fig. 13) is [33]:

$$
t_{x}=t_{v}+\frac{1}{c}(z \cos \alpha+x \sin \alpha)
$$

Investigations have shown that 7 to 9 acquisitions using plane waves produce images with quality comparable to using focused emissions.

\section{LOW-DATA RATE SYSTEMS}

A large part of the cost in an ultrasound system is carried by the specialized beamforming hardware. Modern scanners often use field-programmable gate arrays, which gives certain flexibility and configurability, but are nevertheless relatively difficult to program and modify.

Recent advances in computer hardware makes it tempting to implement beamforming in computer hardware, but the main obstacle is getting the channel data into the system. 


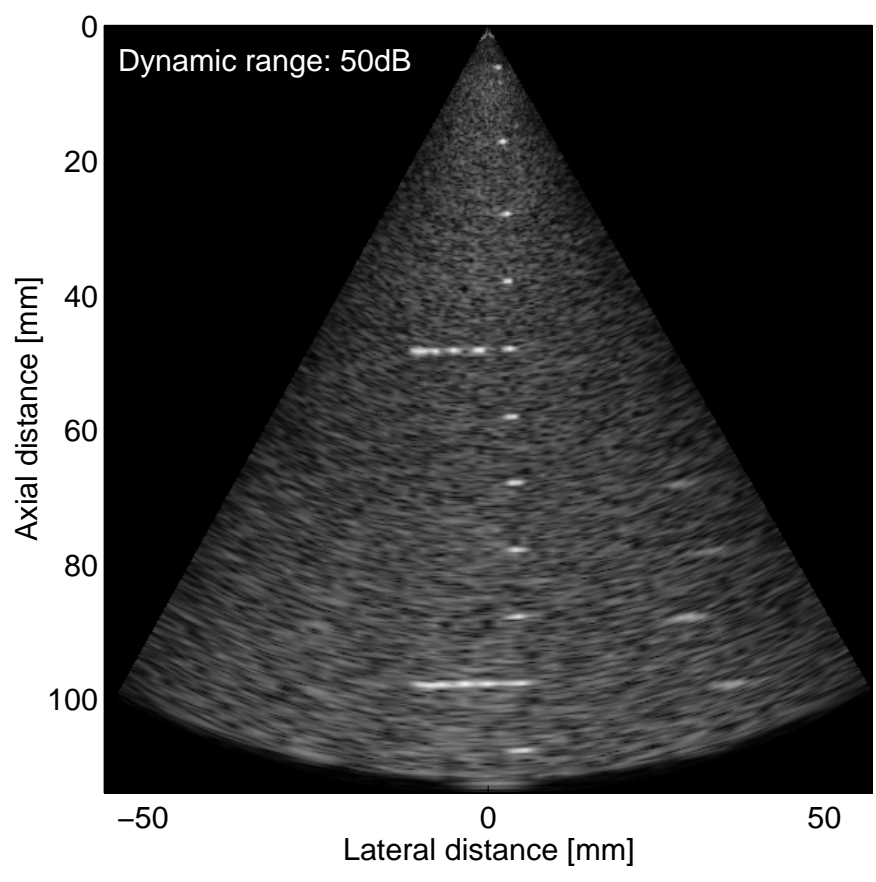

Fig. 14. Sparse synthetic aperture image acquired with 11 transmissions and 64 receive elements (from [20]).

At present a PCI express link has a data rate of about 250 $\mathrm{MB} / \mathrm{s}$ per lane. Sampling, for example, at $20 \mathrm{MHz}, 2$ bytes per sample results in a data rate of $40 \mathrm{MB} / \mathrm{s}$. Data from only 6 channels can be sent via a PCI express link at full speed.

The required data rate can be reduced using synthetic aperture focusing without compromise in the image quality. One method is to use sparse synthetic transmit aperture reducing the number of acquisitions per second, and another is to use two-stage beamforming [9], [13], [14].

\section{A. Sparse transmit aperture}

Figure 14 shows a sparse synthetic aperture image. It uses a $7.5 \mathrm{MHz} \lambda$-pitch linear array transducer, which results in lower angular sensitivity. The number of elements is 64 . The image is comparable to what can be obtained using focused emissions, in spite of the transmit "sparseness".

As described in the previous section, recent investigations using plane wave transmissions have shown that 7 to 9 acquisitions results in an image with quality comparable to conventional scanners that need to perform on the order of 100 to 200 or more acquisition per image. A typical system has a penetration depth of no more than 500 wave lengths. Sampling at 4 times the center frequency gives 4000 samples per acquisition. Designing a system with 128 active channels with a frame rate of 15 to 20 frames / second results in a data rate of

datarate $=$ frames $/ \mathrm{s} \times$ shots $/$ frame $\times$ channels $\times$ bytes $/$ channel

$$
\begin{aligned}
& =20 \times 10 \times 128 \times 8000 \\
& =192(\mathrm{MB} / \mathrm{s}),
\end{aligned}
$$

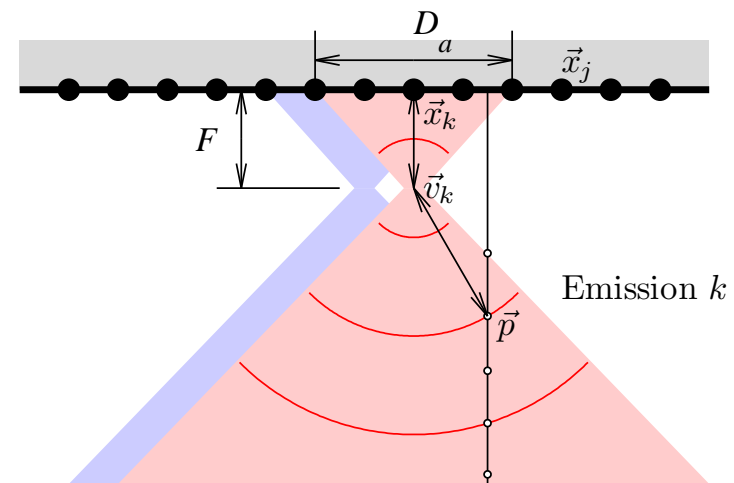

Fig. 15. Two stage beamforming uses fixed focus in the first stage.

which is well within the capabilities of modern hardware. There exist commercial systems that utilize this sparseness.

\section{B. Two stage beamforming}

Two stage beamforming is another way of decreasing the bandwidth to a programmable beamforming unit. The processing is divided in two stages, where the first stage implements fixed focus beamforming. The geometry is shown in Fig. 15. Same delays are applied in transmit and receive and a virtual source $\vec{v}_{k}$ is created at the fixed focus point. Only the elements within the limits of the active aperture $D_{a}$ are used. The delays both in transmit and in receive in the first stage are determined from the geometric distances between the element positions $\vec{x}_{j}$ and the virtual source $\vec{v}_{k}$.

The beamforming in the second stage uses delays calculated from the geometric distances between the virtual point $\vec{v}_{k}$ and the image point $\vec{p}$. The propagation is assumed to be two way - from the virtual point $\vec{v}_{k}$ to the image point $\vec{p}$ and back to the virtual point $\vec{v}_{k}$. The calculation of the propagation time is thus

$$
t=2 \frac{\left|\vec{v}_{k}-\vec{x}_{k}\right|}{c} \pm 2 \frac{\left|\vec{p}-\vec{v}_{k}\right|}{c}
$$

When the point $\vec{p}$ is between the array and the virtual source, the second term in the expression must be subtracted, else added.

Fig. 16 shows the result of two-stage beamforming. Because delays in the second stage compensate for beam diffraction, the synthetic aperture image is focused better.

As a rule of thumb, using two stage beamforming will require creating a number of virtual sources equal to the number of transducer elements. For a 128-element system the data rate will be 128 times less. Designing a system with a 128-element transducer, and a frame rate of 20 frames/s results in a bandwidth of

$$
\begin{aligned}
\text { datarate } & =\text { frames } / \mathrm{s} \times \text { shots } / \text { frame } \times \text { bytes } / \text { virtsrc } \\
& =20 \times 128 \times 8000 \\
& \approx 20(\mathrm{MB} / \mathrm{s})
\end{aligned}
$$

(19) For comparison, USB 2 has a peak data rate of $60 \mathrm{MB} / \mathrm{s}$. 


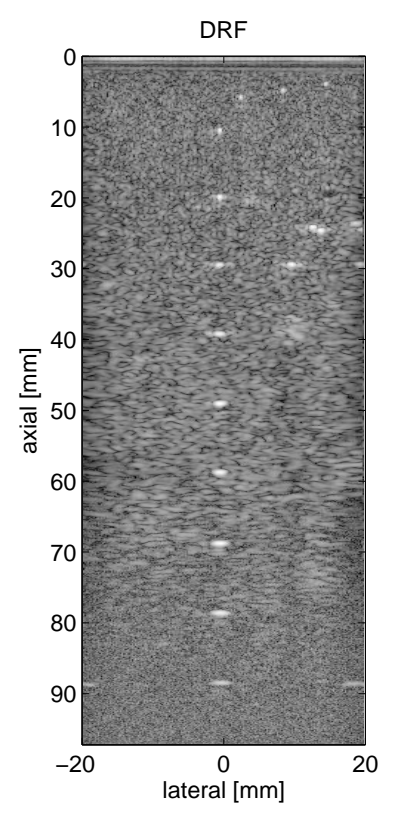

Fig. 16. Conventional dynamically focused image (left) and two-stage sequentially beamformed synthetic aperture image (right). (from [34])

\section{FLOW ESTIMATION}

A major advantage of synthetic aperture focusing is that it allows beamforming a full ultrasound image or a large region in a few transmit/receive events. Flow estimation requires repeated scans of the same spatial location. The pulse repetition frequency is limited by the ability of the estimator to track maximum displacement between measurements. Using an autocorrelation estimator, the displacement of the signal between two measurements should not exceed quarter of a wave length, $\lambda / 4$ [35]. The uncertainty of the estimates is dependent on the ensemble length of the measurements, which typically varies between 4 and 16 .

Because of the inherent capability of synthetic aperture focusing to form full image, it is possible to track the motion in the whole image [1], [36] Consider the example of scanning the carotid artery, which has a maximum flow of about $2 \mathrm{~m} / \mathrm{s}$. The desired pulse-repetition frequency between two pulses is $2.3 \mathrm{kHz}$ or more at a center frequency $f_{0}=7.5 \mathrm{MHz}$.

A typical scan depth is about $4 \mathrm{~cm}$, which allows for $19 \mathrm{kHz}$ pulse repetition frequency. This makes it possible to have a sparse synthetic aperture sequence with 5 to 8 shots per image. The advantage of using synthetic transmit aperture focusing is that it allows for very long ensemble lengths of up to 128 shots per estimate. Furthermore, beams can be formed in the direction of the flow increasing the correlation between data and enabling vector flow estimation.

An analysis of the acquisition process and the nature of the motion artifacts shows that it is possible to generate a highresolution image after every emission [20] and to estimate the flow with very high-precision using interleaved calculation of the correlation function [36].

Fig. 17 illustrates the acquisition process using only trans-

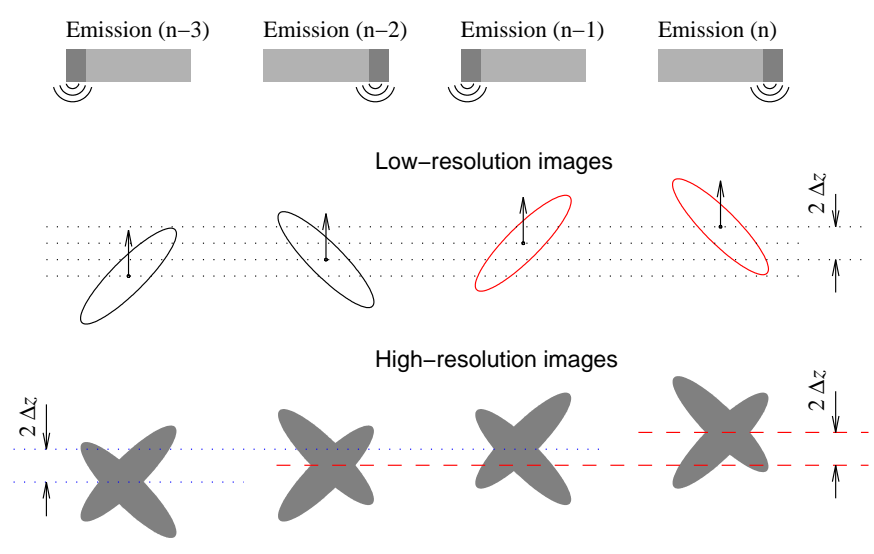

Fig. 17. Illustration of motion artifacts in synthetic aperture imaging (from [1]).

missions across the aperture. Combining emissions $n-3$ with emission $n-2$ can produce a high-resolution image. Combining emission $n-2$ with emission $n-1$ can produce another high-resolution image. Due to the combination of motion with element position and acquisition sequence, the two high-resolution images will not be optimally correlated. A better correlation will exist between the images created with emissions $(n-3)+(n-2)$ and $(n-1)+(n)$. If the motion is constant, the same correlation will exist between images $(n-4)+(n-3)$ and $(n-2)+(n-1)$. These two correlation functions can be averaged to yield a better motion estimate. In essence, for a sparse synthetic aperture using $N_{x m t}$ acquisitions per image, the total acquisition time needed to create a flow image can be decreased $N_{x m t}$ times by using the estimation procedure suggested in [36] without deterioration of image quality and with increased temporal resolution.

Fig. 18 shows an example of an in-vivo synthetic aperture image using cross-correlation velocity estimation and directional beamforming. Despite its smooth appearance, only the raw estimates are shown without any form of post processing. The smoothness is due to high-temporal locality of the estimates and the large size of the data set used in the estimation process.

\section{CONCLUSION}

Synthetic aperture focusing has been adopted by manufacturers of ultrasound equipment as in routine use in commercial scanners. The versatility of the technique makes it useful in a number of applications - from increased image quality in systems using conventional transmit focusing, to highframe-rate, high-performance systems, to low-cost high-image quality scanners.

\section{REFERENCES}

[1] S. I. Nikolov, "Synthetic Aperture Tissue and Flow Ultrasound Imaging," Ph.D. dissertation, Ørsted•DTU, Technical University of Denmark, 2800, Lyngby, Denmark, 2001. 


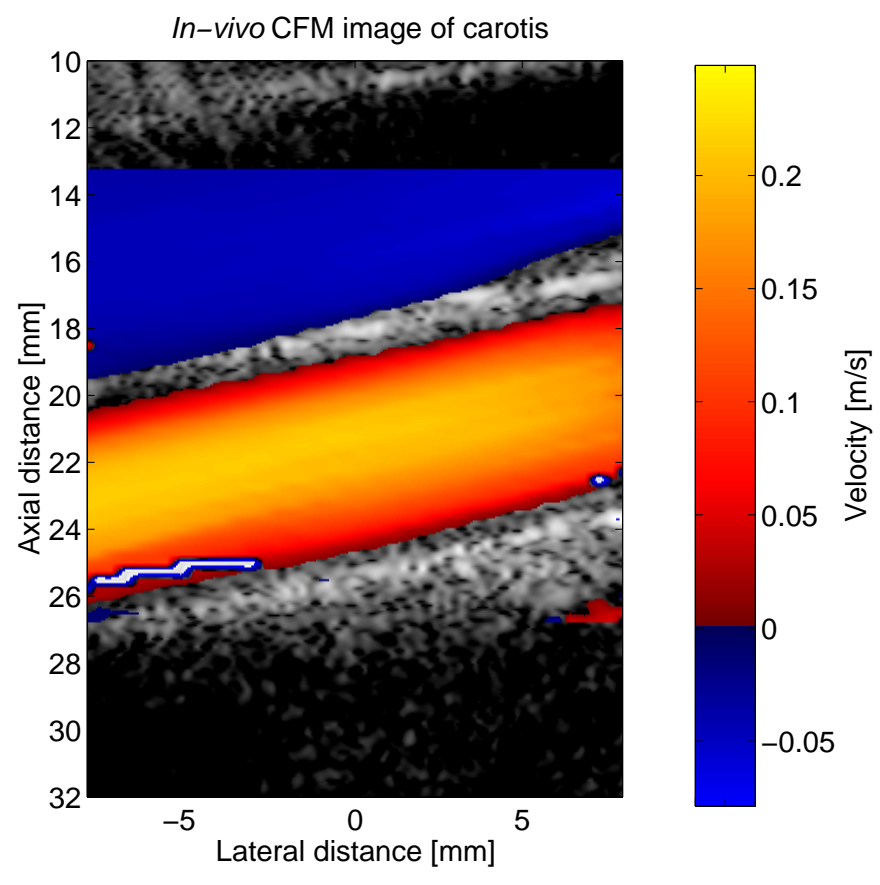

Fig. 18. In vivo color flow map image at a 77-degree flow angle for the jugular vein and carotid artery. The color scale indicates the velocity along the flow direction, where red hues indicate forward flow and blue reverse flow (from [37]).

[2] L. F. Nock and G. E. Trahey, "Synthetic receive aperture imaging with phase correction for motion and for tissue inhomogenities - part I: basic principles," IEEE Trans. Ultrason., Ferroelec., Freq. Contr., vol. 39, pp. 489-495, 1992.

[3] - "Synthetic receive aperture imaging with phase correction for motion and for tissue inhomogenities - part I: basic principles," IEEE Trans. Ultrason., Ferroelec., Freq. Contr., vol. 39, pp. 489-495, 1992.

[4] A. R. Selfridge, G. S. Kino, and B. T. Khuri-Yacub, "A theory for the radiation pattern of a narrow strip acoustic transducer," Appl. Phys. Lett., vol. 37, no. 1, July 1980.

[5] S. Freeman, P. Li, and M. O'Donnel, "Retrospective dynamic transmit focusing," Ultrason. Imaging, vol. 17, pp. 173-196, 1995.

[6] C. Passmann and H. Ermert, "A 100-MHz ultrasound imaging system for dermatologic and ophthalmologic diagnostics," IEEE Trans. Ultrason., Ferroelec., Freq. Contr., vol. 43, pp. 545-552, 1996.

[7] C. H. Frazier and W. D. O'Brien, "Synthetic aperture techniques with a virtual source element," IEEE Trans. Ultrason., Ferroelec., Freq. Contr., vol. 45, pp. 196-207, 1998.

[8] M. H. Bae, M. K. Jeong, T. K. Song, and Y. B. Ahn, "Experimental study of transmit synthetic focusing combined with receive dynamic focusing in B-mode ultrasound imaging systems," in Proc. IEEE Ultrason. Symp. 1999, pp. 1261-1264.

[9] S. I. Nikolov and J. A. Jensen, "Virtual ultrasound sources in highresolution ultrasound imaging," in Proc. SPIE - Progress in biomedical optics and imaging, vol. 3, 2002, pp. 395-405.

[10] C. Bradley, "Retrospective transmit beamformation. ACUSON SC2000 volume imaging ultrasound system," White Paper, Aug 2008.

[11] S. Nikolov, J. A. Jensen, and B. G. Tomov, "Fast parametric beamformer for synthetic aperture imaging," IEEE Trans. Ultrason., Ferroelec., Freq. Contr., vol. 55, no. 8, pp. 1755-1767, 2008

[12] K. Üstüner, "High information rate volumetric ultrasound imaging. ACUSON SC2000 volume imaging ultrasound system," White paper, Aug 2008 .

[13] J. Kortbek, "Synthetic aperture sequential beamforming and other beamforming techniques in ultrasound imaging," Ph.D. dissertation, Ørsted•DTU, Technical University of Denmark, 2800, Lyngby, Denmark, 2007.

[14] J. Kortbek, J. A. Jensen, and K. L. Gammelmark, "Synthetic aperture sequential beamforming," J. Acoust. Soc. Am., p. Submitted, 2009.
[15] S. I. Nikolov and J. A. Jensen, "Comparison between different encoding schemes for synthetic aperture imaging," in Proc. SPIE - Progress in biomedical optics and imaging, vol. 3, 2002, pp. 1-12.

[16] G. R. Lockwood, J. R. Talman, and S. S. Brunke, "Real-time 3-D ultrasound imaging using sparse synthetic aperture beamforming," IEEE Trans. Ultrason., Ferroelec., Freq. Contr., vol. 45, pp. 980-988, 1998.

[17] C. R. Hazard and G. R. Lockwood, "Theoretical assessment of a synthetic aperture beamformer for real-time 3-D imaging," IEEE Trans. Ultrason., Ferroelec., Freq. Contr., vol. 46, pp. 972-980, 1999.

[18] —, "Effects of motion artifacts on a synthetic aperture beamformer for real-time 3d ultrasound," in Proc. IEEE Ultrason. Symp., 1999, pp. 1221-1224.

[19] — , "Effects of motion artifacts on a synthetic aperture beamformer for real-time 3D ultrasound," in Proc. IEEE Ultrason. Symp., 1999, pp. 1221-1224.

[20] S. I. Nikolov, K. Gammelmark, and J. A. Jensen, "Recursive ultrasound imaging," in Proc. IEEE Ultrason. Symp., vol. 2, 1999, pp. 1621-1625.

[21] — " "Velocity estimation using recursive ultrasound imaging and spatially encoded signals," in Proc. IEEE Ultrason. Symp., vol. 2, 2000, pp. 1473-1477.

[22] M. H. Pedersen, K. L. Gammelmark, and J. A. Jensen, "Preliminary in-vivo evaluation of convex array synthetic aperture imaging," in Proc SPIE - Progress in biomedical optics and imaging, 2004, pp. 33-43.

[23] — , "In-vivo evaluation of convex array synthetic aperture imaging," Ultrasound Med. Biol., vol. 33, pp. 37-47, 2007.

[24] J. A. Jensen, O. Holm, L. J. Jensen, H. Bendsen, H. M. Pedersen, K. Salomonsen, J. Hansen, and S. Nikolov, "Experimental ultrasound system for real-time synthetic imaging," in Proc. IEEE Ultrason. Symp. vol. 2, 1999, pp. 1595-1599.

[25] J. A. Jensen, O. Holm, L. J. Jensen, H. Bendsen, S. I. Nikolov, B. G. Tomov, P. Munk, M. Hansen, K. Salomonsen, J. Hansen, K. Gormsen, H. M. Pedersen, and K. L. Gammelmark, "Ultrasound Research Scanner for Real-time Synthetic Aperture Image Acquisition," IEEE Trans. Ultrason., Ferroelec., Freq. Contr., vol. 52 (5), pp. 881-891, May 2005.

[26] J. A. Jensen, M. Hansen, B. G. Tomov, S. I. Nikolov, and H. HoltenLund, "System architecture of an experimental synthetic aperture real time ultrasound system," in Proc. IEEE Ultrason. Symp., Oct. 2007, pp. 636-640.

[27] S. I. Nikolov, J. A. Jensen, and B. G. Tomov, "Recursive delay calculation unit for parametric beamformer," in Proc. SPIE - Progress in biomedical optics and imaging, vol. 6147-13, 2006, pp. 1-12.

[28] — , "Real-time synthetic aperture imaging: opportunities and challanges," in 2006 Fortieth Asilomar Conference on Signals, Systems and Computers, 2006, pp. 1548-1552.

[29] D. Napolitano, C.-H. Chou, G. W. McLaughlin, D. DeBusschere, L. Y. L. Mo, and T.-L. Ji, “Zone-based B-mode imaging," vol. 2, 2003, pp. 25 -28 .

[30] L. Y. L. Mo, T.-L. Ji, C.-H. Chou, D. Napolitano, G. W. McLaughlin, and D. DeBusschere, "Zone-based B-mode imaging," vol. 2, 2003, pp. $29-32$.

[31] M.-K. Jeong, K.-J. Lee, M.-H. Bae, S.-Y. Chang, and S.-B. Gye "Beamforming using the synthetic sinc wave for ultrasonic imaging system,” in Proc. IEEE Ultrason. Symp., vol. 2, 2001, pp. 1539 - 1542.

[32] M. Tanter, J. Bercoff, L. Sandrin, and M. Fink, "Ultrafast Compound Imaging for 2-d Motion Vector Estimation: application to Transient Elastography," IEEE Trans. Ultrason., Ferroelec., Freq. Contr., vol. 49, pp. 1363-1374, 2002.

[33] G. Montaldo, M. Tanter, J. Bercoff, N. Benech, and M. Fink, "Coherent plane-wave compounding for very high-frame rate ultrasonography and transient elastography," IEEE Trans. Ultrason., Ferroelec., Freq. Contr., vol. 56, no. 3, pp. $489-506,2009$.

[34] J. Kortbek, J. A. Jensen, and K. L. Gammelmark, "Synthetic aperture sequential beamforming," in Proc. IEEE Ultrason. Symp., 2008, pp. 966 969.

[35] C. Kasai, K. Namekawa, A. Koyano, and R. Omoto, "Real-Time TwoDimensional Blood Flow Imaging using an Autocorrelation Technique," IEEE Trans. Son. Ultrason., vol. 32, pp. 458-463, 1985.

[36] S. I. Nikolov and J. A. Jensen, "In-vivo Synthetic Aperture Flow Imaging in Medical Ultrasound," IEEE Trans. Ultrason., Ferroelec., Freq. Contr., vol. 50, no. 7, pp. 848-856, 2003.

[37] J. A. Jensen and S. I. Nikolov, "Directional synthetic aperture flow imaging," IEEE Trans. Ultrason., Ferroelec., Freq. Contr., vol. 51, pp. 1107-1118, 2004. 